DOI: 10.20472/IAC.2019.049.014

\author{
HUBERTUS FRANKE \\ Ostfalia University of Applied Sciences, Germany \\ MARTINA HASSELER \\ Ostfalia University of Applied Sciences, Germany \\ DENISE DICK \\ Ostfalia University of Applied Sciences, Germany \\ STEPHANIE KREBS \\ Ostfalia University of Applied Sciences, Germany \\ ANNA LIETZ \\ Ostfalia University of Applied Sciences, Germany \\ SIEGFRIED BUBLITZ \\ Ostfalia University of Applied Sciences, Germany
}

\title{
THE IT-VALUE STREAM MODEL FOR HOSPITAL NETWORKS
}

\begin{abstract}
:
Hospitals, especially in regional settings, often struggle to fulfil all medical demands placed on them due their size and capacity. Thus, it is helpful to cooperate with other hospitals, perhaps in the neighbourhood as a network to exchange resources such as technical equipment or medi-cal staff such as qualified nurses who are in high demand. To solve this problem we can trans-fer some theoretical aspects from other similar situations such as network cooperation in con-nected industry-companies.

This only works by using modern Information Technology based on internet technology and connected IT-Processes. In Germany, the government has implemented an extensive scientific and industrial project called "Industry 4.0 ".

The idea behind "Industry 4.0 " is that all industry processes use digital methods, which are connected but decentralized.

Through the use of Smart technology (computer, smartphone or personal digital assistant - PDA), a Digital Twin representing a complete digital footprint of all units, products and re-sources which are not digital (i.e. human employees) can be created and all digital processes work together (cloud based) to solve problems in real time.

This idea of "Industry 4.0" is useful for Hospital Networks, which often have similar structures. To develop Industry 4.0 for Hospital Networks, we need a special modelling method. An exten-sion of the value stream model (the IT-value stream model) can fulfil this task. In this publica-tion, we demonstrate how an extension of the value stream model can solve the resource prob-lems in Hospital Networks.
\end{abstract}

\section{Keywords:}

Hospital Network, Industry 4.0, Digital Twin, Lean Production, value stream model.

JEL Classification: 112, C63, C51 